\title{
Quantum Field Theory with Electric-Magnetic Duality and Spin-Mass Duality but Without Grand Unification and Supersymmetry
}

\author{
Rainer W. Kühne ${ }^{1}$ \\ Tuckermannstr. 35, Braunschweig, Germany
}

I present a generalization of quantum electrodynamics which includes Dirac magnetic monopoles and the Salam magnetic photon. This quantum electromagnetodynamics has many attractive features. (1) It explains the quantization of electric charge. (2) It describes symmetrized Maxwell equations. (3) It is manifestly covariant. (4) It describes local four-potentials. (5) It avoids the unphysical Dirac string. (6) It predicts a second kind of electromagnetic radiation which can be verified by a tabletop experiment. An effect of this radiation may have been observed by August Kundt in 1885. Furthermore, I discuss a generalization of General Relativity which includes Cartan's torsion. I discuss the mathematical definition, concrete description, and physical meaning of Cartan's torsion. I argue that the electric-magnetic duality of quantum electromagnetodynamics is analogous to the spin-mass duality of Einstein-Cartan theory. A quantum version of this theory requires that the torsion tensor corresponds to a spin-3 boson called tordion which is shown to have a rest mass close to the Planck mass. Moreover I present an empirically satisfied fundamental equation of unified field theory which includes the fundamental constants of electromagnetism and gravity. I conclude with the remark that the concepts presented here require neither Grand Unification nor supersymmetry.

\section{Quantum Electromagnetodynamics}

\subsection{The model}

The quantization of electric charge is well known since the discovery of the proton in 1919 [1]. This remarkable observation remained unexplained within the framework of quantum electrodynamics [2].

Further quantized charges have been established. The group $S U(2)$ of the weak interaction explains the quantization of isospin [3], and the group $S U(3)$ of the strong interaction explains the quantization of colour charge [4].

For this reason we propose the analogy postulate: The quantization of electric charge results from the underlying group structure of the electromagnetic interaction. Hence, we will require neither quantum gravity (electric charge as a topological quantum number [5]), nor spontaneous symmetry breaking (monopoles of soliton type [6]), nor unification with other forces (charge quantization resulting from the group structure underlying grand unified theories) [7].

The electromagnetic angular momentum generated by the Lorentz force in a system consisting of a magnetic monopole and an electric charge is independent of their separation [8]. Angular momen- tum is quantized in units of $\hbar / 2$, where $\hbar=h / 2 \pi$ denotes the reduced Planck constant. This condition can be satisfied only if both electric and magnetic charge are quantized [9]. This is the famous Dirac quantization condition $e g=h$, where $e$ and $g$ denote unit electric and unit magnetic charge.

Magnetic monopoles were discussed long before this finding. The motivation was to describe electric and magnetic fields equivalently by symmetrized Maxwell equations. We will elevate this to the symmetry postulate: The fundamental equations of the electromagnetic interaction describe electric and magnetic charges, electric and magnetic field strengths, and electric and magnetic potentials equivalently.

Dirac [9] was the first to write down these symmetrized Maxwell equations.

Let $J^{\mu}=(P, \mathbf{J})$ denote the electric four-current and $j^{\mu}=(\rho, \mathbf{j})$ the magnetic four-current. The well-known four-potential of the electric photon is $A^{\mu}=(\Phi, \mathbf{A})$. The four-potential of the magnetic photon is $a^{\mu}=(\varphi, \mathbf{a})$. Expressed in three-vectors the symmetrized Maxwell equations read,

$$
\begin{aligned}
\nabla \cdot \mathbf{E} & =P \\
\nabla \cdot \mathbf{B} & =\rho \\
\nabla \times \mathbf{E} & =-\mathbf{j}-\partial_{t} \mathbf{B} \\
\nabla \times \mathbf{B} & =+\mathbf{J}+\partial_{t} \mathbf{E}
\end{aligned}
$$

\footnotetext{
1 kuehne70@gmx.de
} 
and the relations between field strengths and potentials are

$$
\begin{aligned}
& \mathbf{E}=-\nabla \Phi-\partial_{t} \mathbf{A}-\nabla \times \mathbf{a} \\
& \mathbf{B}=-\nabla \varphi-\partial_{t} \mathbf{a}+\nabla \times \mathbf{A}
\end{aligned}
$$

The second four-potential is required not only by the symmetry postulate, but also by the proven impossibility to construct a manifestly covariant one-potential model of quantum electromagnetodynamics.

Although only one of the suggested twopotential models explicitely states the possibility of the existence of a magnetic photon [10], the other two-potential models were eventually considered as two-photon models [11].

Any viable two-photon concept of magnetic monopoles has to satisfy the following conditions.

(i) In the absence of both magnetic charges and the magnetic photon field, the model has to regain the $U(1)$ gauge symmetry of quantum electrodynamics.

(ii) In the absence of both electric charges and the photon field, the symmetry postulate requires the model to yield the $U^{\prime}(1)$ gauge symmetry of quantum magnetodynamics.

(iii) The gauge group has to be Abelian, because the photon carries neither electric nor magnetic charge. Because of the symmetry postulate also the magnetic photon has to be neutral.

(iv) The gauge group may not be simple, because quantum electromagnetodynamics includes the two coupling constants $\alpha_{E}=e^{2} / 4 \pi$ and $\alpha_{M}=$ $g^{2} / 4 \pi$.

The only gauge group that satisfies these four conditions is the group $U(1) \times U^{\prime}(1)$.

A two-photon model has already been suggested by Salam [10]. According to his model the photon couples via vector coupling with leptons and hadrons, but not with monopoles. The magnetic photon couples via vector coupling with monopoles and via tensor coupling with hadrons, but not with leptons.

This model came under severe criticism. Although positron and proton have the same electric charge and no magnetic charge, the model can discriminate them (i.e., leptons and hadrons). For this reason Salam's model does not generate the Lorentz force between electric charge and monopole. As a consequence, it does not satisfy the powerful Dirac quantization condition. For this reason Salam's model was rejected by Taylor [11].

The problem raised by Taylor can be overcome by the following argumentation. Salam considered the tensor coupling of the hadron-monopole system as derivative coupling. This kind of coupling is well known from meson theory where vector mesons are able to interact with baryons via both vector and tensor coupling. However, derivative coupling is possible only where the particles are composite. Hence, Salam's model includes no interaction between lepton and magnetic photon. - We emphasize the correctness of the interpretation of tensor coupling as derivative coupling in meson theory.

To generate the Lorentz force between electric and magnetic charges we have to introduce a new kind of tensor coupling. This is required also, because here we have two kinds of interacting charges (electric and magnetic).

The Coulomb force between two (unit) electric charges is $e^{2} / 4 \pi r^{2}$ Because of the symmetry postulate the magnetic force between two (unit) magnetic charges is $g^{2} / 4 \pi r^{2}$ And the Lorentz force between (unit) electric and (unit) magnetic charge is egv $/ 4 \pi r^{2}$, where $v$ denotes the relative velocity of the two charges.

This suggests the introduction of velocity coupling:

(i) The photon couples via vector coupling with electric charges.

(ii) The magnetic photon couples via vector coupling with magnetic charges.

(iii) The photon couples via tensor coupling with magnetic charges. In contrast to meson theory, however, the $u^{\mu}$ of tensor coupling, $\sigma^{\mu \nu} u_{\nu}$, has to be interpreted as a four-velocity (velocity coupling).

(iv) The magnetic photon couples via tensor coupling (interpreted as velocity coupling instead of derivative coupling) with electric charges.

In the case of the interacting monopole-electric charge system the exchanged boson (either photon or magnetic photon) is virtual and the four-velocity of velocity coupling is the relative four-velocity between the charges.

Charged quanta are required to emit and absorb the same bosons as real (on-mass-shell) particles as those virtual (off-mass-shell) bosons via whom they interact with other charged quanta. This is because the Feynman rules are symmetric with respect to virtual and real particles.

In the case of emission and absorption reactions of real bosons, $u^{\mu}$ cannot be interpreted as a relative four velocity between charged quanta in the initial state, as there is only one charged quantum present. As a consequence, $u^{\mu}$ has to be interpreted as the absolute four-velocity of the initial charged quantum.

In contrast to general belief an absolute rest 
frame is not forbidden. Instead, a number of reasons support its existence (see below).

The aether drift of the Sun was discovered and measured to be $370 \mathrm{~km} / \mathrm{s}$.

\subsection{Formalism}

The Lagrangian for a spin $1 / 2$ fermion field $\Psi$ of rest mass $m_{0}$, electric charge $Q$, and magnetic charge $q$ within an electromagnetic field can be constructed as follows. By using the tensors

$$
\begin{aligned}
F^{\mu \nu} & \equiv \partial^{\mu} A^{\nu}-\partial^{\nu} A^{\mu} \\
f^{\mu \nu} & \equiv \partial^{\mu} a^{\nu}-\partial^{\nu} a^{\mu}
\end{aligned}
$$

the Lagrangian of the Dirac fermion within the electromagnetic field reads,

$$
\begin{aligned}
\mathcal{L}= & -\frac{1}{4} F_{\mu \nu} F^{\mu \nu}-\frac{1}{4} f_{\mu \nu} f^{\mu \nu}+\bar{\Psi} i \gamma^{\mu} \partial_{\mu} \Psi-m_{0} \bar{\Psi} \Psi \\
& -Q \bar{\Psi} \gamma^{\mu} \Psi A_{\mu}-q \bar{\Psi} \gamma^{\mu} \Psi a_{\mu}+Q \bar{\Psi} \gamma^{5} \sigma^{\mu \nu} u_{\nu} \Psi a_{\mu} \\
& +q \bar{\Psi} \gamma^{5} \sigma^{\mu \nu} u_{\nu} \Psi A_{\mu}
\end{aligned}
$$

By using the Euler-Lagrange equations we obtain the Dirac equation

$$
\begin{aligned}
\left(i \gamma^{\mu} \partial_{\mu}-m_{0}\right) \Psi= & \left(Q \gamma^{\mu} A_{\mu}+q \gamma^{\mu} a_{\mu}-Q \gamma^{5} \sigma^{\mu \nu} u_{\nu} a_{\mu}\right. \\
& \left.-q \gamma^{5} \sigma^{\mu \nu} u_{\nu} A_{\mu}\right) \Psi
\end{aligned}
$$

By introducing the four-currents

$$
\begin{aligned}
J^{\mu} & =Q \bar{\Psi} \gamma^{\mu} \Psi-q \bar{\Psi} \gamma^{5} \sigma^{\mu \nu} u_{\nu} \Psi \\
j^{\mu} & =q \bar{\Psi} \gamma^{\mu} \Psi-Q \bar{\Psi} \gamma^{5} \sigma^{\mu \nu} u_{\nu} \Psi
\end{aligned}
$$

the Euler-Lagrange equations yield the two Maxwell equations

$$
\begin{aligned}
J^{\mu} & =\partial_{\nu} F^{\nu \mu}=\partial^{2} A^{\mu}-\partial^{\mu} \partial^{\nu} A_{\nu} \\
j^{\mu} & =\partial_{\nu} f^{\nu \mu}=\partial^{2} a^{\mu}-\partial^{\mu} \partial^{\nu} a_{\nu}
\end{aligned}
$$

Evidently, the two Maxwell equations are invariant under the $U(1) \times U^{\prime}(1)$ gauge transformations

$$
\begin{aligned}
A^{\mu} & \rightarrow A^{\mu}-\partial^{\mu} \Lambda \\
a^{\mu} & \rightarrow a^{\mu}-\partial^{\mu} \lambda
\end{aligned}
$$

Furthermore, the four-currents satisfy the continuity equations

$$
0=\partial_{\mu} J^{\mu}=\partial_{\mu} j^{\mu}
$$

The electric and magnetic field are related to the tensors above by

$$
\begin{aligned}
& E^{i}=F^{i 0}-\frac{1}{2} \varepsilon^{i j k} f_{j k} \\
& B^{i}=f^{i 0}+\frac{1}{2} \varepsilon^{i j k} F_{j k}
\end{aligned}
$$

Finally, the Lorentz force is

$$
\begin{aligned}
K^{\mu}= & Q\left(F^{\mu \nu}+\frac{1}{2} \varepsilon^{\mu \nu \varrho \sigma} f_{\varrho \sigma}\right) u_{\nu} \\
& +q\left(f^{\mu \nu}-\frac{1}{2} \varepsilon^{\mu \nu \varrho \sigma} F_{\varrho \sigma}\right) u_{\nu}
\end{aligned}
$$

where $\varepsilon^{\mu \nu \varrho \sigma}$ denotes the totally antisymmetric tensor. This formula for the Lorentz force is rather trivial for the classical theory. Non-trivial is that this formula can be applied to the quantum field theory. This becomes possible because of the introduction of the velocity coupling which includes a velocity operator and allows the definition of a force operator.

\subsection{Suggested experiment}

This model does not contain any free parameters. Hence, it allows clear and decisive predictions for its verification.

The electric-magnetic duality is:

electric charge - magnetic charge electric current - magnetic current electric conductivity - magnetic conductivity electric field strength — magnetic field strength electric four-potential — magnetic four-potential electric photon - magnetic photon electric field constant - magnetic field constant dielectricity number — magnetic permeability

The absolute frame predicted above gives rise to local physical effects. In a terrestrial laboratory the interaction cross-section of a free magnetic photon (with conventional matter in the terrestrial rest frame) is predicted to be smaller than the one of a free electric photon (= conventional or Einstein photon) of the same energy. The suppression factor is the square of the absolute speed of the laboratory in units of the speed of light. Hence, each reaction that generates electric photons generates also magnetic photons. Magnetic photons are harder to create, to shield, and to absorb than electric photons of the same energy.

The refractive index of an insulator is the square root of the product of the dielectricity number and the magnetic permeability. Therefore it is invariant under a dual transformation. This means that electric and magnetic photon rays are reflected and refracted by insulators in the same way. Optical lenses cannot distinguish between electric and magnetic photon rays.

By contrast, electric and magnetic photon rays are reflected and refracted in a different way by 
metals. This is because electric conductivity and magnetic conductivity determine the reflection of light and they are not identical. The electric conductivity of a metal is several orders larger than the magnetic conductivity.

Light in metal behaves wave-like (polariton, more or less a combination of light wave and sound wave).

The interpretation of the basic equations of quantum electromagnetodynamics is the following. Electric charges can couple to both the fourpotential of the electric photon (via vector coupling) and the four-potential of the magnetic photon (via tensor coupling). So an electric charge generates both an electric four-current density (vector part of the electric four-current density above) and a magnetic four-current density (tensor part of the magnetic four-current density above). According to the Lagrangian, the four-potential of the electric photon can couple only to the electric four-current density, and the four-potential of the magnetic photon can couple only to the magnetic four-current density. The main difference between the vector part and the tensor part of the four-current density is the appearance of the fourvelocity. For emission and absorption processes I interpret this velocity as the absolute velocity of the laboratory (for a terrestrial laboratory: $10^{-3}$ in units of the speed of light). So the magnetic current density is $10^{-3}$ times the electric current density. According to Ohm's law, current density is equal to conductivity times the electromagnetic field. Therefore the magnetic conductivity is $10^{-3}$ times (where $\varepsilon_{0}=1$ ) the electric conductivity of a given conductor in a terrestrial laboratory.

Within a conductor, the penetration depth of light of a given frequency is proportional to the square root of the reciprocal value of the conductivity (for a more precise formula see the following subsection). So I predict that the penetration depth of magnetic photon light is greater than that of electric photon light of the same frequency.

The result would be that in iron (August Kundt experiment, see below) the penetration depth for red light is $7 \mathrm{~nm}$ for electric photon light and 472 $\mathrm{nm}$ for magnetic photon light. In aluminium the penetration depth for green light $(\lambda=532 \mathrm{~nm})$ is $3.35 \mathrm{~nm}$ for electric photon light and $152 \mathrm{~nm}$ for magnetic photon light.

Note that electric conductivity and magnetic conductivity determine the reflection of electric and magnetic photon light, respectively (see equations below). The electric conductivity of a metal is predicted to be larger than the magnetic conduc- tivity. This results in a stronger reflection of electric photon light than magnetic photon light. To give an example: I predict that silver reflects $94 \%$ of the electric photon light, but only $13 \%$ of the magnetic photon light, if green light of the wavelength $532 \mathrm{~nm}$ is used. Therefore the use of mirrors (for reflection) should be avoided for the search for the magnetic photon light.

\subsection{How to verify the magnetic photon rays}

The easiest test to verify/falsify the magnetic photon is to illuminate a metal foil of thickness $100, \ldots, 1000 \mathrm{~nm}$ by a laser beam (or any other bright light source) and to place a detector (avalanche diode or photomultiplier tube) behind the foil. If a single foil is used, then the expected reflection losses are less than $1 \%$. If a laser beam of the visible light is used, then the absorption losses are less than $15 \%$. My model predicts the detected intensity of the radiation to be

$$
f=r(v / c)^{4}
$$

times the intensity that would be detected if the metal foil were removed and the laser beam would directly illuminate the detector. Here

$$
\begin{aligned}
v= & v_{\text {sun }}+v_{\text {earth }} \cos \left(2 \pi t / T_{e}\right) \cos \left(\varphi_{\text {ec }}\right) \\
& +v_{\text {rotation }} \cos \left(2 \pi t / T_{\text {rot }}\right) \cos \left(\varphi_{\text {eq }}\right)
\end{aligned}
$$

is the absolute velocity of the laboratory. The absolute velocity of the Sun as measured by the dipole anisotropy of the cosmic microwave background radiation is

$$
v_{\text {sun }}=(371 \pm 0.5) \mathrm{km} / \mathrm{s}
$$

The mean velocity of the Earth around the Sun is

$$
v_{\text {earth }}=30 \mathrm{~km} / \mathrm{s}
$$

The rotation velocity of the Earth is

$$
v_{\text {rotation }}=0.5 \mathrm{~km} / \mathrm{s} \cos (\varphi)
$$

The latitude of the dipole with respect to the ecliptic is

$$
\varphi_{e c}=15^{\circ}
$$

The latitude of the dipole with respect to the equator (declination) is

$$
\varphi_{e q}=7^{\circ}
$$


The latitude of the laboratory is

$$
\varphi=48^{\circ}
$$

for Strassbourg and Vienna and $\varphi=43^{\circ}$ for Madison. The sidereal year is

$$
T_{e}=365.24 \text { days }
$$

A sidereal day is

$$
T_{\text {rot }}=23 \mathrm{~h} 56 \mathrm{~min}
$$

The zero point of the time, $t=0$, is reached on December 9 at 0:00 local time. The speed of light is denoted by $c$. The factor for losses by reflection and absorption of magnetic photon rays of the visible light for a metal foil of thickness $100, \ldots, 1000 \mathrm{~nm}$ is

$$
r=0.8, \ldots, 1.0
$$

To conclude, quantum electromagnetodynamics predicts the value $f \sim 10^{-12}$.

\subsection{Possible observation of magnetic photon rays}

In Strassbourg in 1885, August Kundt [12] passed sunlight through red glass, a polarizing Nicol, and platinized glass which was covered by an iron layer. The entire experimental setup was placed within a magnetic field. With the naked eye, Kundt measured the Faraday rotation of the polarization plane generated by the transmission of the sunlight through the iron layer. His result was a constant maximum rotation of the polarization plane per length of $418,000^{\circ} / \mathrm{cm}$ or $1^{\circ}$ per $23.9 \mathrm{~nm}$. He verified this result until thicknesses of up to $210 \mathrm{~nm}$ and rotations of up to $9^{\circ}$.

In one case, on a very clear day, he observed the penetrating sunlight for rotations of up to $12^{\circ}$. Unfortunately, he has not given the thickness of this particular iron layer he used. But if his result of a constant maximum rotation per length can be applied, then the corresponding layer thickness was $\sim 290 \mathrm{~nm}$.

Let us recapitulate some classical electrodynamics to determine the behavior of light within iron. (The following equations are nearly identical for electric photon light and magnetic photon light. The only difference is that the electric conductivity has to be replaced by the magnetic conductivity, which is $10^{-3}$ times the electric conductivity in a terrestrial laboratory. There is no interaction between electric current and magnetic current, because in the absence of magnetic charges the vector part of the electric four-current couples only to the four-potential of the electric photon, and the tensor part of the magnetic four-current couples only to the four-potential of the magnetic photon.) The penetration depth of light in a conductor is

$$
\delta=\frac{\lambda}{2 \pi \gamma}
$$

where the wavelength in vacuum can be expressed by its frequency according to $\lambda=1 / \sqrt{\nu^{2} \varepsilon_{0} \mu_{0}}$. The extinction coefficient is

$$
\gamma=\frac{n}{\sqrt{2}}\left[-1+\left(1+\left(\frac{\sigma}{2 \pi \nu \varepsilon_{0} \varepsilon_{r}}\right)^{2}\right)^{1 / 2}\right]^{1 / 2}
$$

where the refractive index is $n=\sqrt{\varepsilon_{r} \mu_{r}}$. For metals we get the very good approximation

$$
\delta \approx\left(\frac{1}{\pi \mu_{0} \mu_{r} \sigma \nu}\right)^{1 / 2}
$$

The specific resistance of iron is

$$
1 / \sigma=8.7 \times 10^{-8} \Omega \mathrm{m}
$$

its permeability is $\mu_{r} \geq 1$. For red light of $\lambda=$ $630 \mathrm{~nm}$ and $\nu=4.8 \times 10^{14} \mathrm{~Hz}$ we get the penetration depth

$$
\delta=6.9 \mathrm{~nm}
$$

Only a small fraction of the sunlight can enter the iron layer. Three effects have to be considered. (i) The red glass allows the penetration of about $\varepsilon_{1} \sim 50 \%$ of the sunlight only. (ii) Only $\varepsilon_{2}=2 / \pi \simeq 64 \%$ of the sunlight can penetrate the polarization filter. (iii) Reflection losses at the surface of the iron layer have to be considered. The refractive index for electric photon light is given by

$$
\bar{n}^{2}=\frac{n^{2}}{2}\left(1+\sqrt{1+\left(\frac{\sigma}{2 \pi \varepsilon_{0} \varepsilon_{r} \nu}\right)^{2}}\right)
$$

For metals we get the very good approximation

$$
\bar{n} \simeq \sqrt{\frac{\mu_{r} \sigma}{4 \pi \varepsilon_{0} \nu}}
$$

The fraction of the sunlight which is not reflected is

$$
\varepsilon_{3}=\frac{2}{1+\bar{n}}=\frac{2}{1+\sqrt{\mu_{r} \sigma /\left(4 \pi \varepsilon_{0} \nu\right)}}
$$


and therefore $\varepsilon_{3} \simeq 0.13$ for the system considered. Taken together, the three effects allow only $\varepsilon_{1} \varepsilon_{2} \varepsilon_{3} \sim 4 \%$ of the sunlight to enter the iron layer.

The detection limit of the naked eye is $10^{-13}$ times the brightness of sunlight provided the light source is pointlike. For an extended source the detection limit depends on the integral and the surface brightness. The detection limit for a source as extended as the Sun $\left(0.5^{\circ}\right.$ diameter $)$ is $l_{d} \sim 10^{-12}$ times the brightness of sunlight. If sunlight is passed through an iron layer (or foil, respectively), then it is detectable with the naked eye only if it has passed not more than

$$
\left(\ln \left(1 / l_{d}\right)+\ln \left(\varepsilon_{1} \varepsilon_{2} \varepsilon_{3}\right)\right) \delta \sim 170 \mathrm{~nm}
$$

Reflection losses by haze in the atmosphere further reduce this value.

Kundt's observation can hardly be explained with classical electrodynamics. Air bubbles within the metal layers cannot explain Kundt's observation, because air does not generate such a large rotation. Impurities, such as glass, which do generate an additional rotation, cannot completely be ruled out as the explanation. However, impurities are not a likely explanation, because Kundt was able to reproduce his observation by using several layers which he examined at various places.

Quantum effects cannot explain the observation, because they decrease the penetration depth, whereas an increment would be required.

The observation may become understandable if Kundt has observed a second kind of electromagnetic radiation, the magnetic photon rays. To learn whether Kundt has indeed observed magnetic photon rays, his experiment has to be repeated.

\subsection{Consequences}

The observation of magnetic photon rays would be a multi-dimensional revolution in physics. Its implications would be far-reaching.

(1) The experiment would provide evidence of a second kind of electromagnetic radiation. The penetration depth of these magnetic photon rays is roughly one million times greater than that ofelectric photon light of the same wavelength. Hence, these new rays may find applications in medicine where X-ray and ultrasonic diagnostics are not useful. X-ray examinations include a high risk of radiation damages, because the examination of teeth requires high intensities of X-rays and genitals are too sensible to radiation damages. Examinations of bones and the brain may also become possible.
(2) The experiment would confirm the existence of a new vector gauge boson, Salam's magnetic photon from 1966 [10]. It has the same quantum numbers as Einstein's electric photon [13], i.e., spin of one, negative parity, zero rest mass, and zero charge. The vanishing rest mass for both the electric and the magnetic photon is required to satisfy the Dirac quantization condition of electric and magnetic charge.

(3) A positive result would provide evidence of an extension of quantum electrodynamics which includes a symmetrization of Maxwell's equations from 1873 [14].

(4) The experiment would provide indirect evidence of Dirac's magnetic monopoles from 1931 and the explanation of the quantization of electric charge [9]. This quantization is known since Rutherford's discovery of the proton in 1919 [1].

(5) My model describes both an electric current and a magnetic current, even in experimental situations which do not include magnetic charges. This new magnetic current has a larger specific resistance in conductors than the electric current. It may find applications in electronics.

(6) Dirac noticed in 1931 that the coupling constant of magnetic monopoles is much greater than unity [9]. This raises new questions concerning the perturbation theory, the renormalizability, and the unitarity of quantum field theories.

(7) The intensity of the magnetic photon rays should depend on the absolute velocity of the laboratory. The existence of the absolute velocity would violate Einstein's relativity principle of special relativity from 1905 [15]. It would be interesting to learn whether there exist further effects of absolute motion.

(8) The supposed non-existence of an absolute rest frame was the only argument against the existence of a luminiferous aether [15]. If the absolute velocity does exist, we have to ask whether aether exists and what its nature is.

(9) When in 1925 Heisenberg introduced quantum mechanics, he argued that motion does not exist in this theory [16]. This view is taken also in the Copenhagen interpretation of quantum mechanics formulated in $1927 / 1928$ by Heisenberg and Bohr [17]. The appearance of a velocity operator in my model challenges this Copenhagen interpretation. Mathematically, the introduction of a velocity (and force) operator means that quantum mechanics has to be described not only by partial but also by ordinary differential equations.

(10) Magnetic photon rays may contribute to our understanding of several astrophysical and 
high energy particle physics phenomena where relativistic absolute velocities appear and where electric and magnetic photon rays are expected to be created in comparable intensities.

(11) Finally, the other interactions may show similar dualities. The new dual partners of the known gauge bosons would be the magnetic photon, the isomagnetic $\mathrm{W}$ - and Z-boson, and the chromomagnetic gluons. In 1999 I argued that the dual partner of the graviton would be the tordion [18]. This boson has a spin of three and is required by Cartan's torsion theory from 1922 [19] which is an extension of Einstein's general relativity from 1915 [20].

\section{Absolute Space and Time}

\subsection{Space and time before General Relativity}

According to Aristotle, the Earth was resting in the centre of the universe. He considered the terrestrial frame as a preferred frame and all motion relative to the Earth as absolute motion. Space and time were absolute [21].

In the days of Galileo the heliocentric model of Copernicus [22] was valid. The Sun was thought to be resting within the centre of the universe and defining a preferred frame. Galileo argued that only relative motion was observed but not absolute motion. However, to fix motion he considered it as necessary to have not only relative motion, but also absolute motion [23].

Newton introduced the mathematical description of Galileo's kinematics. His equations described only relative motion. Absolute motion did not appear in his equations [24].

This inspired Leibniz to suggest that absolute motion is not required by the classical mechanics introduced by Galileo and Newton [25].

Huyghens introduced the wave theory of light. According to his theory, light waves propagate via oscillations of a new medium which consists of very tiny particles, which he named aether particles. He considered the rest frame of the luminiferous aether as a preferred frame [26].

The aether concept reappeared in Maxwell's theory of classical electrodynamics [14]. Faraday [27] unified Coulomb's theory of electricity [28] with Ampère's theory of magnetism [29]. Maxwell unified Faraday's theory with Huyghens' wave theory of light, where in Maxwell's theory light is considered as an oscillating electromagnetic wave which propagates through the luminiferous aether of Huyghens.

We all know that the classical kinematics was replaced by Einstein's Special Relativity [15]. Less known is that Special Relativity is not able to answer several problems that were explained by classical mechanics.

According to the relativity principle of Special Relativity, all inertial frames are equivalent, there is no preferred frame. Absolute motion is not required, only the relative motion between the inertial frames is needed. The postulated absence of an absolute frame prohibits the existence of an aether [15].

According to Special Relativity, each inertial frame has its own relative time. One can infer via the Lorentz transformations [30] on the time of the other inertial frames. Absolute space and time do not exist. Furthermore, space is homogeneous and isotropic, there does not exist any rotational axis of the universe.

It is often believed that the Michelson-Morley experiment [31] confirmed the relativity principle and refuted the existence of a preferred frame. This believe is not correct. In fact, the result of the Michelson-Morley experiment disproved the existence of a preferred frame only if Galilei invariance is assumed. The experiment can be completely explained by using Lorentz invariance alone, the relativity principle is not required.

By the way, the relativity principle is not a phenomenon that belongs solely to Special Relativity. According to Leibniz it can be applied also to classical mechanics.

Einstein's theory of Special Relativity has three problems.

(i) The space of Special Relativity is empty. There are no entities apart from the observers and the observed objects in the inertial frames. By contrast, the space of classical mechanics can be filled with, say, radiation or turbulent fluids.

(ii) Without the concept of an aether Special Relativity can only describe but not explain why electric and magnetic fields oscillate in propagating light waves.

(iii) Special Relativity does not satisfy the equivalence principle [32] of General Relativity, according to which inertial mass and gravitational mass are identical. Special Relativity considers only inertial mass.

Special Relativity is a valid approximation of reality which is appropriate for the description of most of the physical phenomena examined until the beginning of the twenty-first century. However, the macroscopic properties of space and time are better described by General Relativity. 


\subsection{General Relativity: Absolute space and time}

In 1915 Einstein presented the field equations of General Relativity and in 1916 he presented the first comprehensive article on his theory [20]. In a later work he showed an analogy between Maxwell's theory and General Relativity. The solutions of the free Maxwell equations are electromagnetic waves while the solutions of the free Einstein field equations are gravitational waves which propagate on an oscillating metric [33]. As a consequence, Einstein called space the aether of General Relativity [34]. However, even within the framework of General Relativity do electromagnetic waves not propagate through a luminiferous aether.

Einstein applied the field equations of General Relativity on the entire universe [35]. He presented a solution of a homogeneous, isotropic, and static universe, where the space has a positive curvature. This model became known as the Einstein universe. However, de Sitter has shown that the Einstein universe is not stable against density fluctuations [36].

This problem was solved by Friedmann and Lemaître who suggested a homogeneous and isotropic expanding universe where the space is curved [37].

Robertson and Walker presented a metric for a homogeneous and isotropic universe [38]. According to Gödel this metric requires an absolute time [39]. In any homogeneous and isotropic cosmology the Hubble constant [40] and its inverse, the Hubble age of the universe, are absolute and not relative quantities. In the Friedmann-Lemaitre universe there exists a relation between the actual age of the universe and the Hubble age.

According to Bondi and Gold, a preferred motion is given at each point of space by cosmological observations, namely the redshift-distance relation generated by the Hubble effect. It appears isotropic only for a unique rest frame [41].

I argued that the Friedmann-Lemaître universe has a finite age and therefore a finite light cone. The centre-of-mass frame of this Hubble sphere can be regarded as a preferred frame [42].

After the discovery of the cosmic microwave background radiation by Penzias and Wilson [43], it was predicted that it should have a dipole anisotropy generated by the Doppler effect by the Earth's motion. This dipole anisotropy was predicted in accordance with Lorentz invariance [44] and later discovered experimentally [45]. Pee- bles called these experiments aether drift experiments [46].

The preferred frames defined by the RobertsonWalker metric, the Hubble effect, and the cosmic microwave background radiation are probably identical. In this case the absolute motion of the Sun was determined by the dipole anisotropy experiments of the cosmic microwave background radiation to be $(371 \pm 1) \mathrm{km} / \mathrm{s}$.

\subsection{General Relativity: Rotating universe and time travel}

It is well known that planets, stars, and galaxies rotate. So Lanczos and Gamow speculated that the entire universe may rotate and that the rotating universe might have generated the rotation of the galaxies [47].

Gödel was the first to show that a rotating universe is a strict solution of Einstein's field equations for a homogeneous and anisotropic universe. He considered a non-expanding universe and has shown that it allows closed time-like curves, i.e., time-travel. He predicted that the original order of the rotation axes of galaxies was parallel to the universal rotation axis [39].

Raychaudhuri presented a model for an expanding and rotating universe which is a generalization of both the Friedmann-Lemaître universe and the Gödel universe. This cosmology, too, includes closed time-like curves [48].

Possibly, the Raychaudhuri universe did not start from a singularity (big bang), but from a closed time-like curve, i.e., from a time-machine.

Gregory, Thompson, and Tifft discovered that the distribution of the rotation axes for both the spiral and ellipsoid galaxies of the filament-like Perseus-Pisces supercluster is bimodal. One of the peaks is roughly aligned with the major axis of the supercluster while the second peak is roughly $90^{\circ}$ from the first [49]. This anisotropic distribution cannot be explained by conventional models of galaxy-formation. Therefore I suggested that this might be a remnant of the original aligned distribution of galactic rotation axes generated by a rotating universe [50].

A rotating universe with both vorticity and shear would generate an anisotropy of the cosmic microwave background radiation. Collins and Hawking were able to set tight bounds on this effect [51]. However, Korotky and Obukhov showed that the generation of this anisotropy is an effect of shear and not of vorticity alone. So the observed isotropy of the cosmic microwave background radi- 
ation does not contradict the idea of a rotating universe, where the rotation period could be as high as the Hubble age of the universe [52].

There is some discussion whether General Relativity could allow local time-machines. Carter has shown that the Kerr metric [53] of rotating spherical bodies can generate closed time-like curves [54]. This inspired Tipler to investigate a rapidly rotating cylinder with $100 \mathrm{~km}$ length, $15 \mathrm{~km}$ radius, $10^{14} \mathrm{~g} / \mathrm{cm}^{3}$ density, and a rotational speed of $70 \%$ of the speed of light. This object yielded closed time-like curves [55]. However, until now it has not been proved that an observer outside the gravitational field would also see time-travel.

To conclude, General Relativity requires a cosmology which includes a preferred frame, absolute space and time and which may include a rotating universe and time-travel. Such a universe may have originated not from a singularity (big bang), but from a closed time-like curve (time-machine).

\section{Cartan's Torsion}

\subsection{The model}

The torsion tensor can be viewed as the translational field strength. It represents a closure failure of infinitesimal displacements. Infinitesimal parallelograms do not close in a world with torsion. This concept is required by most gauge theories of gravity.

The connection used by general relativity [20] is symmetric. After Eddington [56] suggested to generalize general relativity by introducing an asymmetric connection, Cartan [19] associated angular momentum with the antisymmetric part (= torsion) of an asymmetric connection.

The introduction of quantum mechanics [16] required a quantum theory of gravity whose quantities are no longer classical, but operators. After Yang and Mills [57] suggested to describe quantum field theories by gauge theories, Kibble [58] and Sciama [59] attempted to describe gravity by a gauge theory, where they associated intrinsic spin [60] with Cartan's torsion. The successful description of the quantum field theory of the electroweak interaction by a spontaneously broken gauge theory [3] and the subsequent proof that gauge theories are renormalizable [61] inspired an increasing number of theorists to further develop gauge theories of gravity (for a review see [62]). We will briefly review the arguments for the need for a gauge theory of gravity and the need for a torsion field which we will show to be massive.

Classical electrodynamics and general relativ- ity have well-known analogues. Resting electric charges are the sources of the static Coulomb field and rotating electric charges generate an extra magnetic field and an associated Lorentz force. The field equations of classical electrodynamics are the Maxwell equations, where the matterfree equations describe electromagnetic waves. By analogy, resting masses are the sources of the static gravitational field and rotating masses generate an extra gravitational field associated with the recently discovered [63] Lense-Thirring effect [64]. The field equations of general relativity are the Einstein field equations, where the linearized matter-free equations describe gravitational waves.

But there are also well-known differences. Electrodynamics can be quantized and the Maxwell equations remain the field equations of quantum electrodynamics. Quantization and renormalization are possible, because (in rationalized units) the Lagrangian has dimension - 4 and the coupling constant dimension zero. By contrast, general relativity cannot easily be quantized, because the Lagrangian has dimension - 2 and the coupling constant (Newton's constant) has dimension 2. Hence, a quantum version of general relativity is not renormalizable.

The aim is to find a quantum theory of gravity. Quantum field theories have to yield finite results for all orders of perturbation theory. Infinite contributions have to cancel one another via renormalization. The only quantum field theories yet known to be renormalizable are gauge theories [61].

Hence, the aim is to find a (quantum) gauge field theory of gravity. The first step is to find the appropriate gauge group.

The group underlying special relativity is the Poincarè group. Since general relativity is locally Lorentz invariant, the Poincarè group is a candidate for the gauge group underlying the gauge theory of gravity [62].

The translational part of the Poincarè group is associated with the energy-momentum tensor and therefore with mass. As the metric tensor is of rank two, the gauge boson (graviton) associated with mass has intrinsic spin two.

The rotational part of the Poincarè group is associated with angular momentum [58], [59], [62]. As the torsion tensor [19] is of rank three, its associated gauge boson (tordion [62]) has intrinsic spin three.

The Einstein field equations are symmetric and can describe only spinless matter. This is because intrinsic spin is antisymmetric. The description of 
a Dirac field (which has spin $\hbar / 2$ ) requires the introduction of torsion (which is antisymmetric) [62].

The need for torsion and its association with angular momentum can be seen as follows. The Maxwell equations do not describe electricity and magnetism equivalently. An equivalent description requires the introduction of magnetic charges, where the $U(1)$ group of quantum electrodynamics is extended to the $U(1) \times U^{\prime}(1)$ group. The associated gauge bosons are the Einstein electric photon and the Salam magnetic photon.

By analogy, general relativity does not describe the translational part and the rotational part of the Poincarè group equivalently. An equivalent description requires the introduction of torsion (in analogy to magnetic charge). Furthermore, from the analogy between the Thirring-Lense effect and the Lorentz force we can infer the analogy between angular momentum and magnetic charge. Hence, both torsion and angular momentum are analogous to magnetic charge and therefore associated with one another. The effects of orbital angular momentum are already described by general relativity (Lense-Thirring effect, Kerr metric [53]). Hence, only intrinsic spin can be connected with torsion.

The analogy with isospin suggests that spin is not simply a quantum number, but also the source of a gauge field. Like spin, isospin is described by the group $S U(2)$ [65]. When Heisenberg [65] introduced isospin, he supposed the (weak) nuclear force is an exchange interaction, analogous to the spin exchange interaction with which he and Bethe were able to explain ferromagnetism and antiferromagnetism [66]. Later, the Weinberg-Salam theory [3] has shown that isospin is not simply a quantum number, but also the source of the weak nuclear interaction.

The presented arguments suggest a gauge theory of gravity which requires a gauge boson of spin three that is associated with both torsion and intrinsic spin.

Various gauge theories of gravity which include either massless or massive torsion fields have been suggested. We will now argue for a non-zero rest mass of the tordion.

(i) According to gauge theories charge is conserved if and only if the rest mass of the associated gauge boson is exactly zero. In contrast to total angular momentum, which is the sum of intrinsic spin and orbital angular momentum, intrinsic spin alone is not conserved. Hence, the tordion has to be massive.

(ii) Accelerated charges radiate. In rationalized units the spin $\hbar / 2$ of an electron is greater than its electric charge $e$. If a tordion were massless, then the torsional part of the synchrotron radiation emitted by the electron would be stronger than its electromagnetic part. This would result in a significant difference between the theoretical (according to the standard model) and the actual energy of electrons after acceleration. Such a difference, were it real, is unlikely to have escaped discovery in particle accelerators.

(iii) According to Dirac [9], the electric-magnetic duality (i.e., the introduction of magnetic charges) yields quantized electric and magnetic charges. This result, however, is correct if and only if the electromagnetic field (i.e., both photon and magnetic photon) is massless. By contrast, the spin-mass duality introduced by Kibble [58] and Sciama [59] does not yield quantized charges. Gravitational mass is not quantized. In the linearized approximation of general relativity a massive graviton would change deflection of light by the sun to $3 / 4$ its Einstein (and observed) value [67]. Hence, to agree spin-mass duality and massless graviton with non-quantized mass, we have to assume that the tordion is the massive gauge boson.

(iv) In rationalized units both Fermi's constant [68] of V - A theory [69] and Newton's constant have dimension two. In Weinberg-Salam theory [3], Fermi's constant turns out to be, up to a constant of order unity, the dimensionless coupling constant times the square of the inverse W-boson rest mass. By contrast, Newton's constant is equal to the square of the inverse Planck mass which, however, is not the rest mass of the (massless) graviton. A possibility is to interpret the Planck mass as the rest mass of the second gauge boson of gravity, the tordion.

To conclude, the quantum field theory of gravity is presumably a gauge theory whose underlying group is the Poincarè group. This theory is supposed to include a massive torsion (and associated intrinsic spin) field which breaks the gauge invariance (spontaneously?). The Lagrangian is expected to have the dimension - 4 and the coupling constant should be dimensionless. Finally, the classical, low energy limit has to regain general relativity.

\subsection{What is Cartan's torsion?}

When a four-vector $C^{k}$ is parallely displaced from the four-position $x^{k}$ to $x^{k}+d x^{k}$, then it changes according to the prescription, 


$$
d C^{k}=-\Gamma_{i j}^{k}(x) C^{j} d x^{i}
$$

This is the definition for the position-dependent affine connection $\Gamma_{i j}^{k}$. According to general relativity [20], it has only a symmetric part,

$$
\{\}_{i j}^{k}=\frac{1}{2}\left(\Gamma_{i j}^{k}+\Gamma_{j i}^{k}\right)
$$

which is called "Christoffel symbol." The antisymmetric part of the affine connection is called "Cartan's torsion" [19],

$$
T_{i j}^{k}=\frac{1}{2}\left(\Gamma_{i j}^{k}-\Gamma_{j i}^{k}\right)
$$

According to general relativity, the torsion tensor is zero. The introduction of a nonzero torsion tensor means therefore an extension of general relativity.

Quite remarkably, the torsion tensor transforms as a tensor under local Lorentz transformations [70], whereas the Christoffel symbol does not.

The torsion tensor can be viewed as the translational field strength. It represents a closure failure of infinitesimal displacements. In spacetimes which include torsion, infinitesimal parallelograms do not close.

We know from Einstein's general relativity [20] that gravitational mass is connected with curvature via

$$
G^{i j}=\kappa \Sigma^{i j}
$$

where

$$
G^{i j}=R^{i j}-\frac{1}{2} g^{i j} R_{k}^{k}
$$

is the Einstein tensor, $\Sigma^{i j}$ is the stress-energy (energy-momentum) tensor, $R^{i j}$ is the Ricci tensor, $g^{i j}$ is the metric tensor, $R_{k}^{k}$ is the Ricci scalar, and $\kappa=-8 \pi G / c^{4}$ is the Einstein constant.

Analogously, intrinsic spin is connected with Cartan's torsion via

$$
T^{i j k}=\kappa \tau^{i j k}
$$

where $\tau^{i j k}$ is the spin tensor [62]. The equations above show the analogy between the duality of mass and spin and the duality of curvature and torsion, respectively.

Directly from the definition of the affine connection one obtains the differential equation of autoparallel curves,

$$
\frac{d^{2} x^{k}}{d s^{2}}+\Gamma_{i j}^{k} \frac{d x^{i}}{d s} \frac{d x^{j}}{d s}=0
$$

where the infinitesimal interval $d s$ between $x^{k}$ and $x^{k}+d x^{k}$ is given by

$$
d s^{2}=g_{i j}(x) d x^{i} d x^{j}
$$

Quite remarkably, only the symmetric part of the metric tensor contributes to the square of the infinitesimal interval.

Readers who would like to learn more about the formalism of torsion are invited to read the excellent review, Ref. [62].

\subsection{Why do we need torsion?}

The energy-momentum tensor $\Sigma^{i j}$ of a Dirac field $\Psi$ (spin $1 / 2$ field [9]) is anti-symmetric [71],

$$
\Sigma^{i j}=-\frac{\hbar c}{2}\left[\left(\nabla^{i} \bar{\Psi}\right) \gamma^{j} \Psi-\bar{\Psi} \gamma^{j} \nabla^{i} \Psi\right]
$$

where

$$
\nabla_{i}=\partial_{i}+i e A_{i}
$$

is the covariant derivative. By contrast, the energy-momentum tensor of general relativity [20] is symmetric. In order to couple a spinor field (Dirac field) to a gravitational field, one has to use an energy-momentum tensor which includes antisymmetric parts. Therefore general relativity has to be generalized by the introduction of Cartan's torsion [58].

I have shown that the duality between mass and spin is analogous to the duality between electric charge and magnetic charge [18]. The electricmagnetic duality is,

$$
\begin{aligned}
J^{i} & =\partial_{j} F^{j i} \\
j^{i} & =\partial_{j} f^{j i}
\end{aligned}
$$

where $J^{i}$ is the electric four-current, $j^{i}$ is the magnetic four-current, and the field strength tensors are given by,

$$
\begin{aligned}
F^{j i} & =\partial^{j} A^{i}-\partial^{i} A^{j} \\
f^{j i} & =\partial^{j} a^{i}-\partial^{i} a^{j}
\end{aligned}
$$

where $A^{j}$ is the electric four-potential which corresponds to Einstein's electric photon [13], and $a^{j}$ is the magnetic four-potential which corresponds to Salam's magnetic photon [10].

Comparison of the equations above demonstrates the analogy between the electric-magnetic duality and the mass-spin duality.

The electric-magnetic duality is required to explain the quantization of electric charge [9]. I argued above that magnetic photon radiation may have already been observed by August Kundt in $1885[12]$. 


\subsection{Is there observational evidence for torsion?}

The rotation axes of the galaxies of the PerseusPisces supercluster are aligned. This alignment exists over a distance of at least $40 \mathrm{Mpc}$ (130 million light years) [49]. Such a large alignment cannot be explained within the framework of conventional models of galaxy-formation. Therefore I suggested [50] that this alignment is either a topological defect (torsion wall) or a remnant of the original aligned distribution of galactic rotation axes generated by a rotating universe [39].

\section{Do We Need Grand Unification and Supersymmetry?}

Between 1971 and 1974 supersymmetry has been suggested by several researchers independently [72]. In 1976 researchers suggested a local supersymmetry called supergravity [73]. In 1981 Edward Witten has shown that supersymmetry can solve several shortcomings of Grand Unified theories [74]. In 1984 Michael Green and John Schwarz have shown that string theory and supersymmetry can be combined. This is the superstring theory [75]. In 1995 Edward Witten has shown that the membrane concept can agree the 11-dimensional supergravity with the 10dimensional superstring theory. Both theories are limit cases of an 11-dimensional M-theory [76].

Supersymmetric theories predicted that the elementary particles of the standard theory of particle physics (leptons, quarks, photon, gluons, W- and Z-boson, Higgs boson) have supersymmetric partners. These supersymmetric particles (called neutralinos, photino, gluinos, Winos, Zinos, squarks, and sleptons) were all predicted to have rest masses between 50 and $300 \mathrm{GeV}$.

Now the ATLAS Collaboration of the LHC (Large Hadron Collider) presented data [77] which do not confirm the gluino. It would have been detected if its rest mass were less than $700 \mathrm{GeV}$.

I am not so surprised that signs of light supersymmetric particles have not been detected. I predict that supersymmetry will not be confirmed. My arguments are the following.

(1) The main reason for supersymmetry is that it can explain some shortcomings of minimal Grand Unified Theories, i.e., the mass-hierarchy problem (i.e., the fact that $\mathrm{W}$ - and Z-boson do not have rest masses of $10^{15} \mathrm{GeV}$, although they should have eaten (coupled to) the Higgs bosons of Grand Unification) and the non-observation of the proton decay (lower limit: mean proton lifetime of $10^{33}$ years).

But this argument requires that there is Grand Unification.

In 1997 I suggested a generalization of quantum electrodynamics, called quantum electromagnetodynamics [42]. This theory is based on the gauge group $U(1) \times U^{\prime}(1)$. In contrast to quantum electromagnetodynamics it describes electricity and magnetism as symmetrical as possible. Moreover it explains the quantization of electric charge. It includes electric and magnetic charges (Dirac magnetic monopoles) and two kinds of photon, the conventional Einstein electric photon and the hypothetical Salam magnetic photon. The electricmagnetic duality of this theory reads:

$$
\begin{array}{ll}
\text { electric charge } & \text { - magnetic charge } \\
\text { electric current } & \text { - magnetic current } \\
\text { electric conductivity } & \text { - magnetic conductivity } \\
\text { electric field strength } & \text { - magnetic field strength } \\
\text { electric four-potential } & \text { - magnetic four-potential } \\
\text { electric photon } & \text { - magnetic photon } \\
\text { electric field constant } & \text { - magnetic field constant } \\
\text { dielectricity number } & \text { - magnetic permeability }
\end{array}
$$

Because of the $U(1) \times U^{\prime}(1)$ group structure and the Dirac quantization condition $e g=h$ (unit electric charge times unit magnetic charge is equal to the Planck constant), this theory is hard to agree with Grand Unification. Although a group such as $S U(5) \times S U^{\prime}(5)$ is in principle not impossible.

(2) Another reason for supersymmetry is that it can explain the existence of (anti-symmetrical) fermions in an otherwise symmetrical theory (such as Special Relativity and General Relativity).

However, it has long been known that a generalization of General Relativity which includes antisymmetry is Einstein-Cartan theory. The affine connection of this theory includes not only the nonLorentz invariant symmetrical Christoffel symbol but also the Lorentz invariant anti-symmetrical torsion tensor.

Within the framework of a quantum field theory, the torsion tensor corresponds to a spin-three boson called tordion, which was introduced in 1976 by F. W. Hehl et al. [62].

In 1999 I discussed the properties of the tordion [18]. Moreover I suggested that the electricmagnetic duality is analogous to the mass-spin duality. This analogy reads: 
- electric charge - magnetic charge mass - spin

- electric field constant - magnetic field constant gravitational constant - reduced Planck constant

- electric four-potential - magnetic fourpotential metric tensor - torsion tensor

- electric photon - magnetic photon graviton - tordion

(3) Supersymmetric theories including superstring and $\mathrm{M}$ theory have not much predictive power. For example, so far no one has shown that these theories predict the empirically obvious fundamental equation of unified field theory [78]:

$$
\ln (\kappa c H M)=-1 / \alpha
$$

where $\kappa=8 \pi G / c^{4}$ is the Einstein field constant, $c$ is the speed of light, $H$ is the Hubble constant,
$M=\sqrt{\hbar c / G}$ is the Planck mass, and $\alpha$ is the fine-structure constant. By using the WMAP-5 value [79]

$$
H=(70.5 \pm 1.3) \mathrm{km} /(\mathrm{s} \mathrm{Mpc})
$$

the left-hand side yields

$$
\ln (\kappa c H M)=-137.025(19)
$$

which is within the error bars equal to

$$
-1 / \alpha=-137.035999679(94)
$$

The fundamental equation of unified field theory predicts the Hubble constant to be

$$
H=69.734(4) k m /(s M p c)
$$

\section{References}

[1] E. Rutherford, Phil. Mag. 37, 581 (1919).

[2] S. Tomonaga and J. R. Oppenheimer, Phys. Rev. 74, 224 (1948).

J. Schwinger, Phys. Rev. 74, 1439 (1948).

J. Schwinger, Phys. Rev. 75, 651 (1949).

J. Schwinger, Phys. Rev. 76, 790 (1949).

R. P. Feynman, Phys. Rev. 76, 749 (1949).

R. P. Feynman, Phys. Rev. 76, 769 (1949).

F. J. Dyson, Phys. Rev. 75, 486 (1949).

F. J. Dyson, Phys. Rev. 75, 1736 (1949).

[3] S. Weinberg, Phys. Rev. Lett. 19, 1264 (1967).

A. Salam, Elementary Particle Physics, Ed. N. Svartholm (Almqvist, Stockholm, 1968) p.367.

[4] D. J. Gross and F. Wilczek, Phys. Rev. Lett. 30, 1343 (1973).

H. D. Politzer, Phys. Rev. Lett. 30, 1346 (1973).

S. Weinberg, Phys. Rev. Lett. 31, 494 (1973).

S. Weinberg, Phys. Rev. D 8, 4482 (1973).

H. Fritzsch, M. Gell-Mann, and H. Leutwyler, Phys. Lett. B 47, 365 (1973).

[5] O. Klein, Nature 118, 516 (1926).

[6] G. t'Hooft, Nucl. Phys B 79, 276 (1974).

A. M. Polyakov Sov. Phys. JETP Lett. 20, 194 (1974).

[7] H. Georgi and S. L. Glashow, Phys. Rev. Lett. 32, 438 (1974).

[8] J. J. Thomson, Elements of the Mathematical The- ory of Electricity and Magnetism (Cambridge University Press, 1904).

[9] P. A. M. Dirac, Proc. R. Soc. A 133, 60 (1931).

[10] A. Salam, Phys. Lett. 22, 683 (1966).

[11] J. G. Taylor, Phys. Rev. Lett. 18, 713 (1967).

[12] A. Kundt, S.-B. Preuß. Acad. Wiss. (1885) p. 1055 .

A. Kundt, Wied. Ann. Phys. Chem. 27, 191 (1886).

[13] A. Einstein, Ann. Phys. (Leipzig) 17, 132 (1905).

[14] J. C. Maxwell, A Treatise on Electricity and Magnetism (Clarendon Press, Oxford, 1873).

[15] A. Einstein, Ann. Phys. (Leipzig) 17, 891 (1905).

[16] W. Heisenberg, Z. Phys. 33, 879 (1925).

[17] W. Heisenberg, Z. Phys. 43, 172 (1927).

N. Bohr, Nature 121, 580 (1928)

N. Bohr, Naturwiss. 16, 245 (1928).

N. Bohr, Phys. Rev. 48, 696 (1935).

[18] R. W. Kühne, Int. J. Mod. Phys. A 14, 2531 (1999)

[19] E. Cartan, Compt. Rend. Acad. Sci. 174, 593 (1922).

[20] A. Einstein, S.-B. Preuss. Akad. Wiss., p. 844 (1915).

A. Einstein, Ann. Phys. (Leipzig) 49, 769 (1916).

[21] Aristotle, De caelo (4th century BC).

$[22]$ N. Copernicus, De revolutionibus orbium 
coelestium (1543).

[23] G. Galilei, Discorsi e dimostrazioni matematiche intorno a due nuove scienze attenenti alla meccanica ed i movimente locali (Leida, Elsevier, 1638).

[24] I. Newton, Philosophiae naturalis principia mathematica (London, 1687).

[25] G. W. Leibniz, Third letter to S. Clarke (1716).

[26] C. Huyghens, Traité de la lumière (1690).

[27] M. Faraday, Experimental Researches in Electricity, Vol. I (London, Taylor and Francis, 1839).

M. Faraday, Experimental Researches in Electricity, Vol. II (London, Richard and John Edward Taylor, 1844).

M. Faraday, Experimental Researches in Electricity, Vol. III (London, Taylor and Francis, 1855).

[28] C. A. Coulomb, Hist. Mém. l'Acad. R. Sci., p. 569 (1785).

C. A. Coulomb, Hist. Mém. l'Acad. R. Sci., p. 578 (1785).

C. A. Coulomb, Hist. Mém. l'Acad. R. Sci., p. 612 (1785).

C. A. Coulomb, Hist. Mém. l'Acad. R. Sci., p. 67 (1786).

[29] A.-M. Ampère, Ann. Chim. Phys. 15, 59 (1820). A.-M. Ampère, Ann. Chim. Phys. 15, 170 (1820).

[30] J. Larmor, Aether and Matter (Cambridge, University Press, 1900).

H. A. Lorentz, Proc. R. Acad. Amsterdam 6, 809 (1904).

[31] A. A. Michelson, Am. J. Sci. 22, 120 (1881).

A. A. Michelson and E. W. Morley, Am. J. Sci. 34, 333 (1887).

[32] A. Einstein, Ann. Phys. (Leipzig) 38, 355 (1912).

[33] A. Einstein, S.-B. Preuss. Akad. Wiss., p. 154 (1918).

[34] A. Einstein, Äther und Relativitätstheorie (Berlin, Springer-Verlag, 1920).

[35] A. Einstein, S.-B. Preuss. Akad. Wiss., p. 142 (1917).

[36] W. de Sitter, Konin. Ned. Akad. Wetenschappen 19, 1217 (1917)

[37] A. Friedmann, Z. Phys. 10, 377 (1922).

A. Friedmann, Z. Phys. 21, 326 (1924).

G. Lemaître, Ann. Soc. Sci. Brux. 47, 49 (1927).

[38] H. P. Robertson, Astrophys. J. 82, 284 (1935).

A. G. Walker, Proc. London Math. Soc. 42, 90 (1936).

[39] K. Gödel, Rev. Mod. Phys. 21, 447 (1949).

[40] E. P. Hubble, Proc. Nat. Acad. Sci. 15, 168 (1929).

[41] H. Bondi and T. Gold, Nature 169, 146 (1952).

[42] R. W. Kühne, Mod. Phys. Lett. A 12, 3153 (1997).

[43] A. A. Penzias and R. W. Wilson, Astrophys. J. 142, 419 (1965).

[44] P. J. E. Peebles and D. T. Wilkinson, Phys. Rev. 174, 2168 (1968).

R. N. Bracewell and E. K. Conklin, Nature 219, 1343 (1968).

[45] G. F. Smoot, M. V. Gorenstein, and R. A. Muller, Phys. Rev. Lett. 39, 898 (1977).
[46] P. J. E. Peebles, Physical Cosmology (Princeton, University Press, 1971).

[47] C. Lanczos, Z. Phys. 21, 73 (1924).

G. Gamow, Nature 158, 549 (1946).

[48] A. Raychaudhuri, Phys. Rev. 98, 1123 (1955).

[49] S. A. Gregory, L. A. Thompson, and W. G. Tifft, Astrophys. J. 243, 411 (1981).

[50] R. W. Kühne, Mod. Phys. Lett. A 12, 2473 (1997).

[51] S. W. Hawking, Mon. Not. R. Astron. Soc. 142, 129 (1969).

C. B. Collins and S. W. Hawking, Mon. Not. R. Astron. Soc. 162, 307 (1973).

[52] V. A. Korotky and Yu. N. Obukhov, Sov. Phys. JETP 72, 11 (1991)

Yu. N. Obukhov, Gen. Relativ. Grav. 24, 121 (1992).

S. Carneiro and G. A. Mena Marugán, Phys. Rev. D 64, 083502 (2001).

[53] R. P. Kerr, Phys. Rev. Lett. 11, 237 (1963).

[54] B. Carter, Phys. Rev. 174, 1559 (1968).

[55] F. J. Tipler, Phys. Rev. D 9, 2203 (1974).

[56] A. S. Eddington, Proc. Roy. Soc. A 99, 104 (1921).

[57] C. N. Yang and R. L. Mills, Phys. Rev. 96, 191 (1954).

[58] T. W. B. Kibble, J. Math. Phys. 2, 212 (1961).

[59] D. W. Sciama, Rev. Mod. Phys. 36, 463 (1964).

[60] G. E. Uhlenbeck and S. Goudsmit, Naturwissenschaften 13, 953 (1925).

[61] B. W. Lee and J. Zinn-Justin, Phys. Rev. D 5, 3121 (1972).

B. W. Lee and J. Zinn-Justin, Phys. Rev. D 5, 3137 (1972).

B. W. Lee and J. Zinn-Justin, Phys. Rev. D 5, 3155 (1972).

G. t'Hooft and M. Veltman, Nucl. Phys. B 44, 189 (1972).

G. t'Hooft and M. Veltman, Nucl. Phys. B 50, 318 (1972).

[62] F. W. Hehl et al., Rev. Mod. Phys. 48, 393 (1976).

[63] I. Ciufolini et al., Science 279, 2100 (1998).

[64] J. Lense and H.Thirring, Phys. Z. 19, 156 (1918).

[65] W. Heisenberg, Z. Phys. 77, 1 (1932).

[66] W. Heisenberg, Z. Phys. 49, 619 (1928).

H. A. Bethe, Z. Phys. 71, 205 (1931).

[67] H. Van Dam and M. Veltman, Nucl. Phys. B 22, 397 (1970).

V. I. Zakharov, JETP Lett. 12, 312 (1970).

L. H. Ford and H. Van Dam, Nucl. Phys. B 169, 126 (1980).

D. G. Boulware and S. Deser, Phys. Rev. D 6, 3368 (1972)

H. Van Dam and M. Veltman, Gen. Rel. Grav. 3, 215 (1972).

[68] E. Fermi, Z. Phys. 88, 161 (1934).

[69] R. P. Feynman and M. Gell-Mann, Phys. Rev. 109, 193 (1958).

E. C. G. Sudarshan and R. E. Marshak, Phys. Rev. 109, 1860 (1958).

J. Sakurai, Nuovo Cim. 7, 649 (1958). 
[70] A. Einstein, S.-B. Preuss. Acad. Wiss. (1929) p. 2.

[71] O. Costa de Beauregard, C. R. Acad. Sci. 214, 904 (1942).

O. Costa de Beauregard, J. Math. Pures Appl. 22, 85 (1943).

[72] P. Ramond, Phys. Rev. D 3, 2415 (1971).

Yu. A. Gol'fand and E. P. Likhtman, JETP Lett. 13, 323 (1971).

D. Volkov and V. P. Akulov, Phys. Lett. B 46, 109 (1973).

J. Wess and B. Zumino, Nucl. Phys. B 70, 39 (1974).

[73] S. Deser and B. Zumino, Phys. Lett. B 62, 335 (1976).

D. Z. Freedman, P. van Nieuwenhuizen and S. Ferrara, Phys. Rev. D 13, 3214 (1976).

[74] E. Witten, Nucl. Phys. B 188, 513 (1981).
[75] M. B. Green and J. H. Schwarz, Phys. Lett. B 149, 117 (1984).

[76] E. Witten, Nucl. Phys. B 443, 85 (1995).

[77] ATLAS Collaboration, G. Aad et al., Phys. Rev. Lett. 106, 131802 (2011).

[78] R. W. Kühne, Mod. Phys. Lett. A 27, 1917 (1999).

[79] E. Komatsu et al., Astrophys. J. Suppl. 180, 330 (2009).
Received: 7 January, 2011

Accepted: 24 August, 2011 